\title{
Evaluación de la resistencia a antibióticos en enterobacterias aisladas de aguas contaminadas.
}

\section{Carta al Editor}

José A. Rivera-Tapia ${ }^{1}$, Lilia Cedillo-Ramírez ${ }^{1,2}$.

${ }^{1}$ Centro de Investigaciones Microbiológicas, ${ }^{2}$ Posgrado en Ciencias Ambientales del Instituto de Ciencias de la Enemérita Universidad Autónoma de Puebla, Puebla, Puebla, México.

Durante los últimos años, el problema de la contaminación de los ríos se ha agravado debido al incremento incesante de las poblaciones y por la demanda de servicios públicos. Los ríos Atoyac y Alseseca en el Estado de Puebla, México, tienen una mala calidad de sus aguas, representando potencialmented un problema de salud pública, ya que éstos tienen microorganismos potencialmente patógenos (1).

Las fuentes de contaminación más importantes son las descargas industriales y municipales, constituyendo los principales abastecedores de aguas negras a dichos ríos. Estas descargas presentan contaminación química, física y biológica. Dentro de la contaminación biológica destaca la presencia de microorganismos, principalmente enterobacterias (1).

En el periodo de septiembre-diciembre del 2002, se realizó un diagnóstico de enterobacterias en la descarga municipal del boulevard las Torres del río Alseseca. A las muestras colectadas se les realizó la prueba del número más probable; además del aislamiento e identificación se evaluó su patrón de susceptibilidad a antibióticos, por medio de la técnica de Kirby Bauer. Los datos mostraron que el río Alseseca rebasa la densidad microbiana de coliformes totales $1100 \mathrm{NMP} / 100 \mathrm{~mL}$. Se aislaron un total de 100 bacterias, de las cuales el $91 \%$ fueron enterobacterias y el $9 \%$ otros microorganismos no fermentadores. Escherichia coli fue el más representativo de los aislamientos, con 44\%, Klebsiella 17\%, Serratia 13\% y Enterobacter con $10 \%$ (2).

El trabajo "Efecto de las aguas residuales hospitalarias sobre los patrones de resistencia a antibióticos de Escherichia coli y Aromonas sp" (3), incluyó aguas de una quebrada aledaña a un hospital en San José Costa Rica, estudiándose los patrones de resistencia a antibióticos en E. coli y Aeromonas $\mathrm{sp}$, mostrando que ambos grupos de microorganismos son resistentes principalmente a eritromicina, ampicilina y amoxicilina (3).

Por su parte, el trabajo que realizamos, involucrando muestras de agua del río Alseseca, Municipio de Puebla, México, el cual recibe

Solicitud de sobretiros: M. en C. José A. Rivera-Tapia, Centro de Investigaciones Microbiológicas, Instituto de Ciencias, Benemérita Universidad Autónoma de Puebla. Edificio 76 Complejo de Ciencias, Ciudad Universitaria, C.P. 72570, Puebla, Puebla, México.

Correo electrónico: jart70@yahoo.com

Recibido el 3/Enero/2005. Aceptado para publicación el 17/Enero/2005.

Este artículo está disponible en http://www.uady.mx/sitios/biomedic/revbiomed/pdf/rb051629.pdf

Vol.16/No.2/Abril-Junio, 2005 
JA Rivera-Tapia, L Cedillo-Ramírez.

Cuadro 1

Porcentajes totales de resistencia a antibióticos en los diversos aislamientos.

\begin{tabular}{lcccccccc}
\hline & CB & AM & CTX & SXT & CL & AK & GE & NET \\
E. coli & 90 & $30^{*}$ & $15^{*}$ & $23^{*}$ & $15^{*}$ & $0^{*}$ & $0^{*}$ & 0 \\
Klebsiella & 87 & 67 & 34 & 0 & 14 & 0 & 0 & 0 \\
Serratia & 91 & 50 & 41 & 0 & 0 & 0 & 0 & 0 \\
Enterobacter & 89 & 77 & 55 & 22 & 22 & 22 & 22 & 0 \\
\hline
\end{tabular}

$\mathrm{CB}=$ carbenicilina, $\mathrm{AM}=$ ampicilina, $\mathrm{CTX}=$ cefotaxima, $\mathrm{SXT}=$ trimetropim,

$\mathrm{Cl}=$ clorafenicol, $\mathrm{AK}=$ amikacina, $\mathrm{GE}=$ gentamicina, $\mathrm{NET}=$ netilmicina

* Valores comparables con los reportados por Tzoc et al, (2004)

principalmente descargas industriales y municipales, mostró un comportamiento parecido en lo que respecta a susceptibilidad a antibiótico (cuadro 1). Al comparar ambos trabajos, se observa que para E. coli, en el trabajo presentado por Tzoc y col., (3) reportan la siguiente resistencia: $5.5 \%$ para amikacina, $57 \%$ para ampicilina, $45 \%$ para amoxicilina, 34\% para trimetropim, $14 \%$ para gentamicina, $14 \%$ para clorafenicol y $12 \%$ para cefotaxima. Es importante señalar que las variaciones observadas en la comparación de los datos reportados por uno y otro trabajo está en función a la fuente del muestreo. Sin embargo, es importante resaltar que aún siendo de diferentes zonas geográficas, la resistencia a antibióticos parece tener un patrón especifico en $E$. coli.

En los países en vías de desarrollo, la aparición de microorganismos resistentes a antibióticos, se facilita debido al mayor número de casos de enfermedades entericas y al uso de antibióticos que no son prescritos por los médicos. Además debe considerarse la relación medio ambiente-salud en los estudios referentes a problemas de resistencia a antibióticos entre patógenos, la emergencia y reemergencia de enfermedades infecciosas a nivel mundial. Otro aspecto que hay que considerar, es el papel que juega en estos mecanismos el cambio climático $(4,5)$. De tal forma, para evitar dicho problema de resistencia a antibióticos, es necesario como medida preventiva, el uso prudente de los mismos en medicina humana y medicina veterinaria.

Palabras clave: resistencia antimicrobiana, enterobacterias, contaminación, Puebla, México.

\section{REFERENCIAS}

1.- Rivera-Tapia JA, Rosales PM, Román MC, Muñoz ZG. Diagnóstico de enterobacteria en muestras de agua provenientes de ríos. En: Rocha GC, Cedillo RL, Lopez OF, editores. Métodos de la investigación en ambiente y salud. Puebla: Publicaciones Especiales de la Benemérita Universidad Autónoma de Puebla, México; 2001. p. 37-49.

2.- Guzmán CM. Diagnóstico de enterobacterias durante el periodo septiembre-diciembre 2002 en la descarga del Boulevard las Torres del río Alseseca en el Municipio de Puebla. Tesis. Benemérita Universidad Autónoma de Puebla, 2004.

3.- Tzoc E, Arias ML, Valiente C. Efecto de las aguas residuales hospitalarias sobre los patrones de resistencia a antibióticos de Escherichia coli y Aeromonas sp. Rev Biomed 2004; 15: 165-72.

4.- Solórzano-Santos F, Miranda-Novales MG. Resistencia de bacterias respiratorias y enterobacterias a antibióticos. Salud Pública Mex 1998; 40: 510-6.

5.- Rivera-Tapia JA. Environment and health. An Med Asoc Med Hosp ABC 2003; 48: 223-7.

\section{Revista Biomédica}

